\title{
Automated Tool Based on Deep Learning to Assess Voltage Dips Validity: Integration in the QuEEN MV network Monitoring System
}

\author{
M. Zanoni, R. Chiumeo, L. Tenti, M. Volta \\ Ricerca sul Sistema Energetico - RSE S.p.A. \\ Via Rubattino 54, 20134, Milano, Italy \\ Phone/Fax number:+00390239921
}

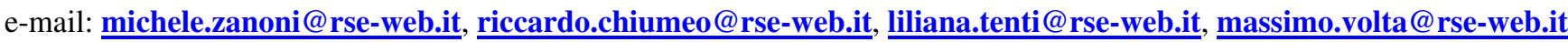

\begin{abstract}
This paper presents the development of an automated tool called QuEEN PyService, aimed to the extraction of events voltage signals from the QuEEN distribution network monitoring system database, for advanced Power Quality analysis. The application has allowed the integration of the DELFI classifier (DEep Learning for False voltage dips Identification), recently developed by RSE, making it possible for the first time the intensive validation of the latter on a large number of voltage dips. Thanks to this tool, a comparison between the performance of DELFI and those of an older criterion based on the $2^{\text {nd }}$ voltage harmonic measurement has been performed using data recorded by 61 measurement units in the period 2015-2020 The analysis has been focused on traditional PQ voltage dips counting indices as $\mathrm{N}_{2 a}$ e $\mathrm{N}_{3 b}$. Results show that the usage of the DELFI classifier increases the $\mathrm{N}_{2 \mathrm{a}}$ and the $\mathrm{N}_{3 \mathrm{~b}}$ by respectively the $20.6 \%$ and $38.8 \%$ with respect to the QuEEN criterion.
\end{abstract}

Key words. deep learning, voltage dip, automatic detection, power quality, monitoring system.

\section{Introduction}

In the last years, the distribution grid development has to face demanding requirements for achieving the innovative features and capabilities of the smart grid paradigm. In this context power quality (PQ) is a key-task [1]. In fact, the increasing penetration of renewable energy power plants, power electronic devices, and (in the near future) plug-in electrical vehicles charging stations has boosted considerably the harmonic pollution level and transient phenomena effects in the distribution grid. For these reasons the demand for widespread monitoring systems is crucial for properly handling PQ phenomena [2].

RSE manages the research monitoring system QuEEN (Qualità dell'Energia Elettrica) [3] operating in the Italian MV distribution grid since 2006. The system consists of nearby 400 measurement devices installed in the primary substations uniformly distributed throughout Italy. Each measurement unit (MU) perform different power quality measurements (e.g. flicker, short voltage interruptions, voltage dips (VD) and swells, etc.) as prescribed by the IEC-61000-4-30 [4]. The monitoring system is compliant with the standard CEI EN50160 [5] so that it automatically records VDs from the voltage rms values in terms of residual voltage (RV) and dip duration (DD).

On the other hand, the Italian distribution grid is still characterized by a significant presence of isolated neutral systems ( $25 \%$ of MV busbar are operated in this way). In this configuration, voltage transformers (VT) saturation can occur during particular events (i.e. single line-to-ground faults) leading to "false" voltage dips.

In order to handle this problem a criterion, based on the detection of the $2^{\text {nd }}$ harmonic component has been implemented in QuEEN. The criterion, developed by RSE in cooperation with the Politecnico di Torino [6], [7] is able to identify "false" voltage dips with a global performance of $87 \%$. Indeed, the $2^{\text {nd }}$ harmonic criterion has not a Boolean answer, as in some undefined cases as very short false voltage dips or multistage events, it is not able to identify the event type (true or false).

In order to enhance the QuEEN functionalities, RSE has recently developed a different approach, based on Deep Learning (DL) algorithms in which the "jpg image" of the sequence of the rms voltage values associated to a voltage dip is adopted as input data (DELFI - DEep Learning for False voltage dips Identification), in order to try to solve the undefined cases. In this way the classifier provides anyway a Boolean answer [8]. The performances achieved on a small set of 130 events was of $95 \%$ [9].

Based on these promising results, this paper presents a tool that integrate the DELFI application, feeding it with voltage signals extracted directly from the QuEEN monitoring system database. This application, called QuEEN PyService, has been developed in Python language and it provides the following automated functions: (i) data reading of all voltage dips recorded by the QuEEN system MUs; (ii) generation of archive containing all the images representing the voltage dips sequences of RMS values; (iii) classification of the events in terms of their validity by the Deep Learning classifier.

Using this tool, it has been possible to compare the performance achieved by the new classifier with those of the QuEEN criterion on a broad statistical sample consisting of the voltage dips recorded by 61 MUs during 
the period from 2015 to 2020 . For each of these events, the DELFI application has been applied.

The analysis has been performed by evaluating the traditional PQ counting indices $\mathrm{N}_{2 \mathrm{a}}$ and $\mathrm{N}_{3 \mathrm{~b}}$ from the voltage dips classified as true, one after the other, by the two criteria. These indices, counting the events occurring under the immunity curves for Class 2 and Class 3 equipment, increase with the number of severe events. The PQ analysis results show that the adoption of the DELFI classifier leads to an increase of these "severity" indices.

\section{QuEEN PyService Architecture}

The QuEEN PyService is an automated tool developed by RSE in Python environment. The application interacts directly with the QuEEN database. The developed functions are mainly focused on advanced analysis of voltage dips, even if the architecture can potentially manage other types of PQ parameters stored in the QuEEN database. In particular, the main purpose has been to integrate a DL classifier for the recognition of the validity of the voltage dips developed by RSE and described in [8] and [9]. The architecture of the application is shown in Fig. 1.

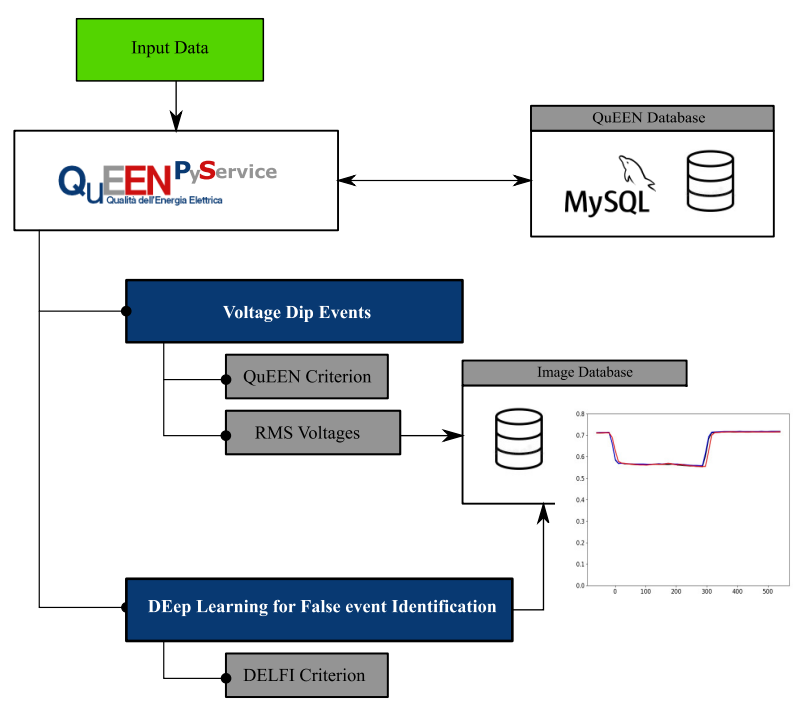

Fig. 1. Queen PyService Architecture.

The application queries the system by selecting a time period and a list of MUs. The following functions are then performed:

- reading of all the voltage dips recorded by the selected MUs over the chosen period;

- generation of an archive of images representing the rms values associated with each event, however it has been classified (true, false or undefined) by the $2^{\text {nd }}$ harmonic criterion;

- application of the DELFI classifier to each voltage dips recorded.

In the following, the architecture of the application and the different functions implemented are presented, in more detail. The system has been developed in a scalable way, so that it can be subsequently integrated into the QuEEN system itself or into other dedicated platforms.

\section{A. Interaction with QuEEN Database}

Once received the input data, the application directly establishes a connection to the server in which all the data collected by the MUs are stored in the QuEEN database. The QuEEN PyService, queries the database through MySQL language getting the following information for each voltage dip:

- MU identification code;

- $\quad$ start and stop event time stamps;

- QuEEN validity criterion ${ }^{1}$ outcome: a field that identifies the outcome of the algorithm based on the $2^{\text {nd }}$ harmonic evaluation, implemented in the MUs firmware [6],[7], that can assume the following values:

$$
\begin{array}{ll}
\circ & \text { true event }(\mathrm{T}) ; \\
\circ & \text { not defined event (ND); } \\
\circ & \text { false event (F); }
\end{array}
$$

- Residual Voltage (RV);

- Dip Duration (DD).

Moreover, for each recorded voltage dip, QuEEN PyService generates an image representing the rms values sequence of the line-to-line voltages recorded by the MU. The DELFI application is then activated providing the images previously generated as input data for the DELFI criterion based on Deep Learning algorithms. The implemented DL algorithm is presented in the next section.

\section{B. DELFI Application}

The DELFI (Deep Learning for False Events Identification) [10],[11], is an application developed by RSE based on Deep Learning (DL) algorithms [12] for the recognition of false voltage dips recorded by the QuEEN monitoring system. In fact, it is well known that, in distribution networks operated with isolated neutral, the saturation of voltage transformers can cause a false voltage drop at the output of the transducer due to the zero-sequence voltage component evolution during faults to ground extinction.

The identification of false events reminds a typical "Machine Learning" (ML) and "Pattern Recognition" problem. Indeed, the voltage waveforms associated to such events show typical aspects (the camel humps and Doge's hat pattern) that should be recognized by a properly trained Deep Learning (DL) classifier. These typical patterns are highlighted also in the rms sequence associated to the voltage waveforms. The voltage waveforms and the associated rms sequence values of a typical example of a false event are respectively shown in Fig. 2 and Fig. 3.

\footnotetext{
1 The criterion relies on 2 parameters (a threshold for the $2^{\text {nd }}$ harmonic level and the minimum number of consecutive exceeding of the $2^{\text {nd }}$ harmonic threshold) and it has to be applied independently to all the three phases.
} 


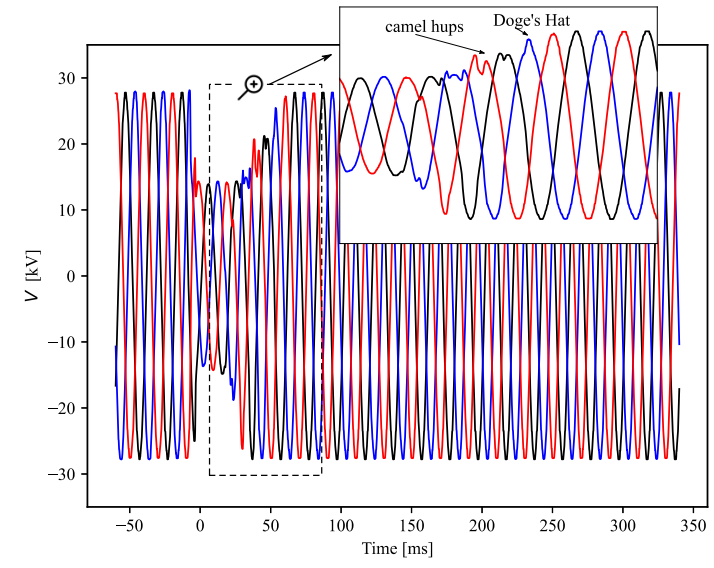

Fig. 2. False event: voltage waveforms with the characteristic camel humps and Doge's hat patterns.

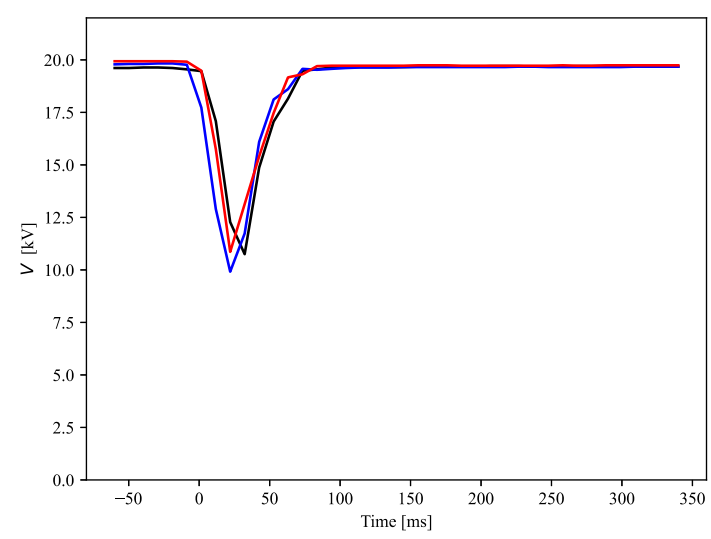

Fig. 3. False event: rms voltage values.

In [9], the two types of DL algorithms developed by RSE are presented, which use two different type of input data for voltage dips: the voltage waveforms and the rms sequences. In both cases the input data actually consist of a set of "jpg" images of the above-mentioned signals associated to false, true and true + false $e^{2}$ voltage dips. The models implemented and optimized are based on a Convolutional Neural Network (CNN) [12] with only one hidden layer. The developed models characteristics can be summarized as follows:

- $80 \%-10 \%-10 \%$ input data partitioning into the Training Set, the Development Set and the Test Set respectively;

- Bayesian optimization (BO) [13],[14] adoption to assess the model hyper-parameters;

- $\quad$ softmax algorithm use as classifier.

The results presented in [9], referring to a small Test Set of 130 events, show that the model with rms sequences as input data achieves the best performance ( $\sim 95 \%$ overall accuracy).

For this reason, in this work, that particular model has been chosen for the integration in QuEEN PyService in order to assure the availability of a DL classifier in the

\footnotetext{
${ }^{2}$ Multistage events, which usually include both true and false events, due to poly-phase grounded faults.
}

QuEEN monitoring system. The architecture of the adopted model is shown in Fig. 4 while the hyperparameters obtained by $\mathrm{BO}$ performed in [9] are reported in Table I.

Table I. - DELFI Hyper-parameters

\begin{tabular}{|l|c|}
\hline $\mathrm{CNN}^{\circ}$ of layers & 1 \\
\hline $\mathrm{N}^{\circ}$ of kernel matrix & 63 \\
\hline mini-batch size & 33 \\
\hline learning rate & $1.2 \mathrm{e}-05$ \\
\hline maxEpoch & 20 \\
\hline
\end{tabular}
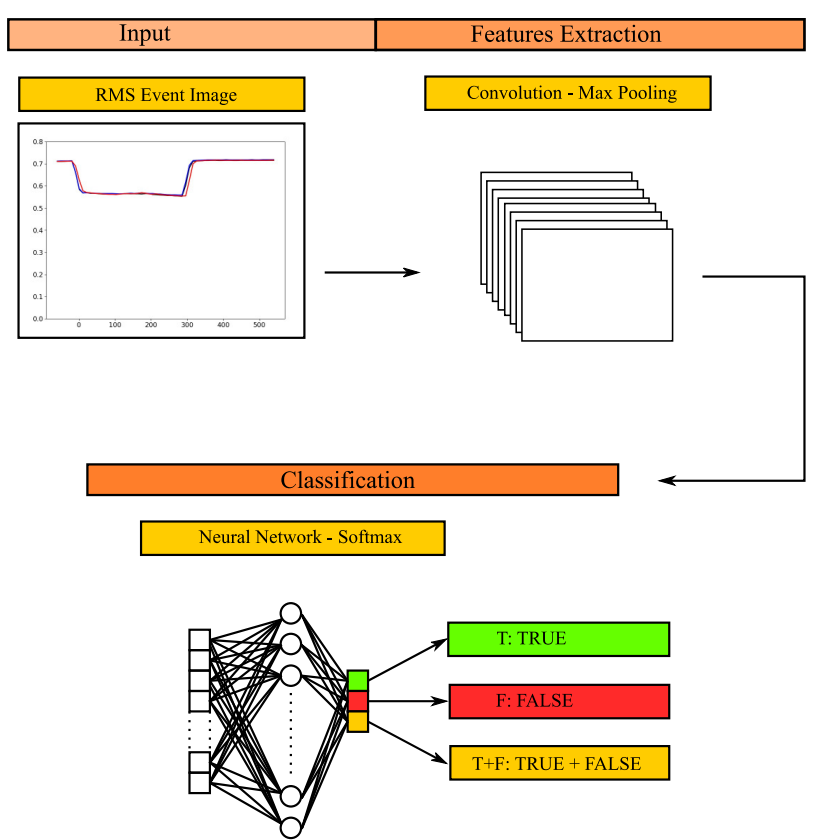

Fig. 4. DELFI Architecture.

\section{Power Quality Analysis on Voltage Dips}

The main goal of the QuEEN PyService application is to perform advance PQ analysis on the events stored in the QuEEN database. In particular, the main focus of this work is to compare the effects that the DL classifier could have on the voltage dips characterization in terms of severity compared to those shown by the QuEEN criterion.

It is well known that voltage dips severity is determined by the events main characteristics, DD and RV, as prescribed by CEI EN 50160 standard [5] and reported in Fig. 5. Through these characteristics, different indices and classification methods have been proposed [5], [15] Thanks to these parameters, an evaluation of the disturbances impact on the "industrial" user can be performed together with the attribution of responsibility between DSO (Distribution System Operator) and User.

The most intelligible parameters are the simple counting indices $\mathrm{N}_{2 \mathrm{a}}$ and $\mathrm{N}_{3 b}$; these indices respectively count, without any kind of weighting, the events that fall below the immunity zones for Class 2 [16] (dot-orange line in Fig. 5) and for Class 3 equipment [17] (continuous-red line in Fig. 5). In particular, the $\mathrm{N}_{3 \mathrm{~b}}$ is most significant as it counts the number of voltage dips below the so-called responsibility curve between DSO and User and could be 
a possible "candidate" for MV network regulation purposes.

\begin{tabular}{|c|c|c|c|c|c|}
\hline \multirow{2}{*}{$\begin{array}{c}\text { Residual Voltage } \\
u[\%]\end{array}$} & \multicolumn{5}{|c|}{ Duration $t[\mathrm{~ms}]$} \\
\hline & $10 \leq t \leq 200$ & $200<t \leq 500$ & $500<t \leq 1000$ & $1000<t \leq 5000$ & $5000<t \leq 60000$ \\
\hline $90>u \geq 80$ & CELLA1 & CELLA2 & CELLA3 & CELLA4 & CELLA5 \\
\hline $80>u \geq 70$ & CELL B1 & CELL B2 & CELL B3 & CELL B4 & CELL B5 \\
\hline $70>u \geq 40$ & CELL C1 & CELL C2 & CELL C3 & CELL C4 & CELL C5 \\
\hline $40>u \geq 5$ & CELL D1 & CELL D2 & CELL D3 & CELL D4 & CELL D5 \\
\hline $5>u$ & CELL X1 & CELL X2 & CELL X3 & CELL X4 & CELL X5 \\
\hline
\end{tabular}

Fig. 5. CEI EN 50160 Voltage Dips classification and thresholds for $\mathrm{N}_{2 \mathrm{a}}$ (dotted-orange line) and $\mathrm{N}_{3 b}$ (continuos-red line) severe events counting indices.

QuEEN PyService computes $\mathrm{N}_{2 \mathrm{a}}$ and $\mathrm{N}_{3 \mathrm{~b}}$ first of all for each true event in accordance with the corresponding algorithm. In fact, these indices are evaluated considering both the criteria implemented in the application, namely the $2^{\text {nd }}$ harmonic and DELFI classifier.

On the other hand, the MUs during a specific period, may be out of service due to faults or maintenance. For this reason, in order to have a significant statistical analysis, it is important to define the Equivalent Measurement Point (EMP) for the selected monitoring period (typically one year). This parameter provides an evaluation of the number of measurement devices actually functioning in the selected time period and it can be computed as reported in (1):

$$
E M P=\frac{\sum_{N^{\circ} \mathrm{MU}} N^{\circ} \text { actual operation week }}{\sum_{N^{\circ} \mathrm{MU}} N^{\circ} \text { theoretical operation week }} N^{\circ} \mathrm{MU}
$$

Therefore, the $\mathrm{N}_{2 a}$ e $\mathrm{N}_{3 b}$ indices have been computed with respect to the EMP (relative indices) providing the level of severe events in the network as the number of severe voltage dips per measurement point $\left(\mathrm{N}^{\circ} / \mathrm{EMP}\right)^{3}$.

\section{The Results}

The analysis of voltage dips has been performed on 61 MUs over the last six years from the 2015 to 2020. Each voltage dip presents two validity classifications: the former performed with the QuEEN criterion and the latter with the DELFI classifier. Voltage dips occurred simultaneously with the relays trip of High Voltage (HV) line distance protections (HV origin events) have been removed from the results ${ }^{4}$.

First of all, let us consider results concerning the QuEEN criterion: the voltage dips trends with respect to the three event types are shown in Fig. 6 and listed in percentage values in Table II: the total number of voltage dips fluctuates over the years ranging from a minimum value of 5760 events, in 2020, to a maximum value of 9136 events, in 2019. It is worth to highlight that not defined events are not classified events namely when $2^{\text {nd }}$ harmonic

\footnotetext{
3 The use of this parameter, rather than the number of MV bus bars monitored by the system makes the PQ analysis more precise.

${ }^{4}$ This information has been read automatically by the QuEEN PyService from the QuEEN Database.
}

component criterion fails, and its occurrence is far to be neglected.

On the other hand, the percentage ratios between the different categories are maintained: averagely, the $78 \%$ are classified as true events while false and not defined events represent respectively the $12 \%$ and $11 \%$ of the total number of voltage dips.

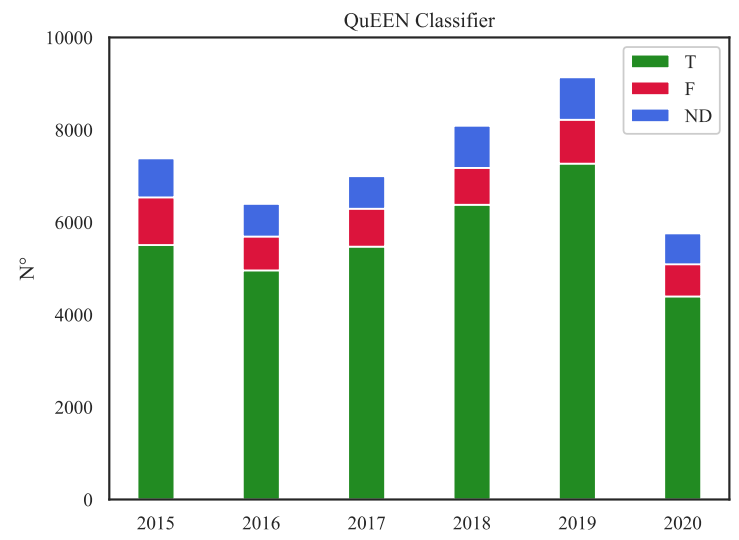

Fig. 6. Voltage dips trends from 2015 to 2020: partition into the three QuEEN criterion categories.

Table II. - Trend of QuEEN criterion events types percentages.

\begin{tabular}{|c|c|c|c|}
\hline \multirow{2}{*}{ Categories [\%] } & \multicolumn{3}{|c|}{ QuEEN } \\
\cline { 2 - 4 } & $\boldsymbol{T}$ & $\boldsymbol{F}$ & $\boldsymbol{N D}$ \\
\hline $\mathbf{2 0 1 5}$ & $74.6 \%$ & $14.0 \%$ & $11.4 \%$ \\
\hline $\mathbf{2 0 1 6}$ & $77.5 \%$ & $11.5 \%$ & $11.0 \%$ \\
\hline $\mathbf{2 0 1 7}$ & $78.2 \%$ & $11.7 \%$ & $10.1 \%$ \\
\hline $\mathbf{2 0 1 8}$ & $78.9 \%$ & $9.9 \%$ & $11.3 \%$ \\
\hline $\mathbf{2 0 1 9}$ & $79.6 \%$ & $10.4 \%$ & $10.0 \%$ \\
\hline $\mathbf{2 0 2 0}$ & $76.3 \%$ & $12.1 \%$ & $11.6 \%$ \\
\hline
\end{tabular}

Now let us consider, the results achieved by the DELFI classifier: the annual trends are reported in Fig. 7 while results, expressed in percentage values, are listed in Table III.

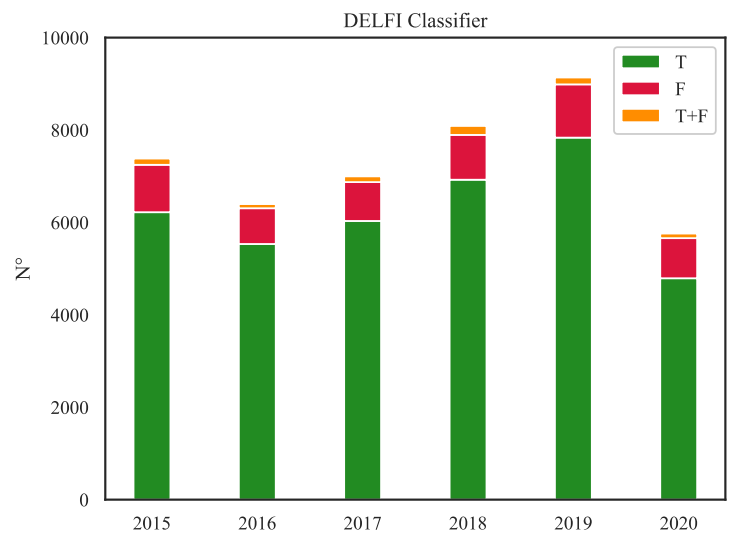

Fig. 7. Voltage dips trends from 2015 to 2020: partition into the three DELFI classifier categories. 
Table III. - Trend of DELFI classifier categories percentages.

\begin{tabular}{|c|c|c|c|}
\hline \multirow{2}{*}{ Categories [\%] } & \multicolumn{3}{|c|}{ DELFI } \\
\cline { 2 - 4 } & $\boldsymbol{T}$ & $\boldsymbol{F}$ & $\boldsymbol{T + F}$ \\
\hline $\mathbf{2 0 1 5}$ & $84.3 \%$ & $13.9 \%$ & $1.8 \%$ \\
\hline $\mathbf{2 0 1 6}$ & $86.5 \%$ & $12.1 \%$ & $1.4 \%$ \\
\hline $\mathbf{2 0 1 7}$ & $86.2 \%$ & $12.0 \%$ & $1.8 \%$ \\
\hline $\mathbf{2 0 1 8}$ & $85.6 \%$ & $12.0 \%$ & $2.4 \%$ \\
\hline $\mathbf{2 0 1 9}$ & $85.8 \%$ & $12.6 \%$ & $1.6 \%$ \\
\hline $\mathbf{2 0 2 0}$ & $83.2 \%$ & $15.1 \%$ & $1.7 \%$ \\
\hline
\end{tabular}

Comparing the DELFI classifications with the QuEEN criterion ones, it can be stated that the number of detected true events is significantly increased reaching almost the $85 \%$ of the total number of voltage dips monitored. On the other hand, the number of false events remains almost the same passing from the 12\% (reached by the QuEEN criterion) to nearby the $13 \%$; moreover, the number of true +false event represents only the $1.8 \%$. From these preliminary results it can be stated that the not defined events monitored by QuEEN are mostly classified as true events by the DELFI algorithm.

Now let us consider the PQ indices presented in Section 3. The $\mathrm{N}_{2 \mathrm{a}}$ and $\mathrm{N}_{3 \mathrm{~b}}$ trends over the years are shown in Fig. 8 for both the classification criteria; the indices are expressed in terms of number of events per equivalent measurement point $\left(\mathrm{N}^{\circ} / \mathrm{EMP}\right)$

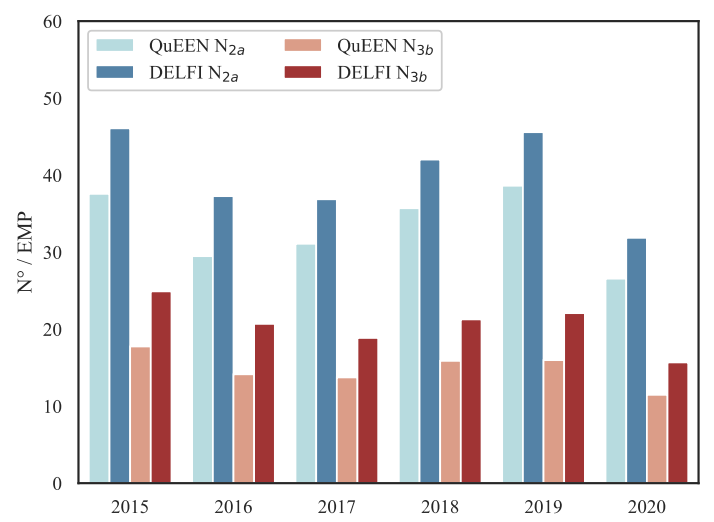

Fig. 8. $\mathrm{N}_{2 \mathrm{a}}$ e $\mathrm{N}_{3 \mathrm{~b}}$ annual trends: comparison between QuEEN criterion and DELFI classifier.

By looking at the achieved results, the following conclusions can be drawn:

- referring at first to true events by the QuEEN criterion, $\mathrm{N}_{2 \mathrm{a}}$ has an average value over the considered period of $33.2 \mathrm{~N}^{\circ} / \mathrm{EMP}$ while $\mathrm{N}_{3 \mathrm{~b}}$ reaches an average value of $14.8 \mathrm{~N}^{\circ} / \mathrm{EMP}$;

- the application of the DELFI classifier considerably increases PQ indices: the average values for both $\mathrm{N}_{2 \mathrm{a}}$ and $\mathrm{N}_{3 \mathrm{~b}}$ reach respectively $40.0 \mathrm{~N}^{\circ} / \mathrm{EMP}$ and $20.6 \mathrm{~N}^{\circ} / \mathrm{EMP}$.

This means that a considerable number of events categorized as not defined or false by the QuEEN criterion but classified as true by the DELFI algorithm, significantly contributes to $\mathrm{N}_{2 \mathrm{a}}$ and $\mathrm{N}_{3 \mathrm{~b}}$ evaluation.
In order to evaluate the percentage difference between the two classification methodologies, the following parameter has been defined:

$$
\Delta=\frac{\left.\mathrm{N}_{x}\right|_{\text {DELFI }}-\left.\mathrm{N}_{x}\right|_{\text {QuEEN }}}{\left.\mathrm{N}_{x}\right|_{\text {QuEEN }}} \cdot 100 \%
$$

where $x$ represents the considered index. Results are reported in Table IV for each considered year together with $\mathrm{N}_{2 \mathrm{a}}$ e $\mathrm{N}_{3 b}$ values.

Table IV. - $\mathrm{N}_{2 \mathrm{a}}$ and $\mathrm{N}_{3 \mathrm{~b}}$ comparison: QuEEN vs. DELFI.

\begin{tabular}{|c|c|c|c|c|c|c|}
\hline \multirow{2}{*}{} & \multicolumn{3}{|c|}{$\mathrm{N}_{2 \mathrm{a}}$} & \multicolumn{3}{c|}{$\mathrm{N}_{3 \mathrm{~b}}$} \\
\cline { 2 - 7 } & QuEEN & DELFI & $\Delta[\%]$ & QuEEN & DELFI & $\Delta[\%]$ \\
\hline $\mathbf{2 0 1 5}$ & 37.6 & 46.1 & +22.7 & 17.8 & 24.9 & +40.3 \\
\hline $\mathbf{2 0 1 6}$ & 29.5 & 37.3 & +26.5 & 14.1 & 20.7 & +46.3 \\
\hline $\mathbf{2 0 1 7}$ & 31.1 & 36.9 & +18.6 & 13.7 & 18.9 & +37.3 \\
\hline $\mathbf{2 0 1 8}$ & 35.7 & 42.0 & +17.7 & 15.9 & 21.3 & +33.9 \\
\hline $\mathbf{2 0 1 9}$ & 38.6 & 45.6 & +18.0 & 16.0 & 22.1 & +38.2 \\
\hline $\mathbf{2 0 2 0}$ & 26.6 & 31.9 & +22.7 & 11.5 & 15.7 & +40.3 \\
\hline
\end{tabular}

By looking at the listed results, it can be noticed that there is a systematic trend: $\mathrm{N}_{2 \mathrm{a}}$ evaluated by the DELFI classifier is always higher, on average by $20.6 \%$, than the same index calculated according to the QuEEN criterion, while the $\mathrm{N}_{3 \mathrm{~b}}$ increase is equal to $38.8 \%$. This means that a considerable number of events classified as true only by the DELFI algorithm contribute to both indices with a prevalent impact on $\mathrm{N}_{3 \mathrm{~b}}$. Therefore, the adoption of a more accurate validity classifier has a not negligible impact on the evaluation of the PQ indices $\mathrm{N}_{2 \mathrm{a}}$ and $\mathrm{N}_{3 \mathrm{~b}}$.

\section{Conclusion}

In this paper the development of an automated tool, called QuEEN PyService, has been presented, aimed to make advanced voltage dips analysis available in the QuEEN MV network monitoring system. First of all this application has allowed the integration of the DELFI classifier, based on Deep Learning techniques and using voltage rms sequences images as input data. The DELFI classifier is aimed to assess the validity of voltage dips and clean their statistics from voltage drops due to the measurement transformers saturation. The classifier provides always a Boolean classification (true, false and true + false). This allows a more accurate classification of the events respect to that achieved by the $2^{\text {nd }}$ harmonic component criterion implemented in the QuEEN system, as it provides a certain number of not defined events.

Thanks to QuEEN PyService, the voltage dips recorded by 61 MUs between 2015 and 2020 have been considered: based on these data PQ analysis have been focused on a $\mathrm{N}_{2 \mathrm{a}}$ and $\mathrm{N}_{3 \mathrm{~b}}$ more accurate evaluation. Results show that the not defined cases by the QuEEN criterion are mostly classified as true by the DELFI classifier. Those events contribute a lot to both $\mathrm{N}_{2 \mathrm{a}}$ and $\mathrm{N}_{3 \mathrm{~b}}$. As to the DELFI classifier, it boosts the abovementioned indices respectively of the $20.6 \%$ and the $38.8 \%$ with respect to those evaluated by the QuEEN criterion. 


\section{Acknowledgement}

This work has been financed by the Research Fund for the Italian Electrical System in compliance with the Decree of Minister of Economic Development April 16, 2018.

\section{References}

[1] M. H. J. Bollen et al., "Power Quality Concerns in Implementing Smart Distribution-Grid Applications," IEEE Trans. Smart Grid, vol. 8, no. 1, pp. 391-399, Jan. 2017.

[2] CIGRE/CIRED JWG C4.112, "Guidelines For Power Quality Monitoring - Measurement Locations, Processing And Presentation Of Data," TB, October 2014.

[3] http://queen.rse-web.it/

[4] IEC 61000-4-30, "Electromagnetic Compatibility (EMC)Part 4-30: Testing and Measurement Techniques-Power Quality Measurement Methods,”, 2015.

[5] CEI EN 50160, "Voltage characteristics of electricity supplied by public distribution networks,", 2010.

[6] R. Chiumeo, M. De Nigris, C.Gandolfi, L. Garbero, L. Tenti, E. Carpaneto, "Implementation of a new method for an improved voltage dip evaluation by the Italian Power Quality Monitoring System in Presence of VT Saturation Effects," in $14^{\text {th }}$ International Conference on Renewable Energies and Power Quality ICREPQ'10, Granada, 2010.

[7] R. Chiumeo, C.Gandolfi, L. Garbero, L. Tenti, E. Carpaneto, "Power Quality," ISBN (10): 1-4438-9493-1, ISBN (13): 978-1-4438-9493-7, Part II, Chapter 7, pg. 102114, Editor Mircea Ion Buzdugan, Manuel Perez Donsion, Cambridge Scholar Publishing, 2016.

[8] R. Chiumeo, H. Shadmehr, L. Tenti, "A machine learning based tool for voltage dips classification," $29^{\text {th }}$ International Conference \& Exhibition on Electricity Distribution, CIRED 2019, paper 0985, 2019

[9] L. Tenti, R. Chiumeo, M. Zanoni and H. Shadmehr, "Deep Learning to Assess Voltage Dips Validity," 2020 AEIT International Annual Conference (AEIT), Catania, Italy, 2020, pp. 1-4.

[10] F. Malegori, H. Shadmehr, L.Tenti, M. Volta, F. Zanon, "Impatto sull'utente dei disturbi di PQ e automatizzazione dei metodi per la sua valutazione". RSE Report $\mathrm{n}^{\circ}$ 17006319, Chapter 2, 2017 (in Italian).

[11] H. Shadmehr, "Analisi per la definizione di un modello di validazione dei buchi di tensione e la caratterizzazione dell loro origine con tecniche di Deep Learning", RSE Report ${ }^{\circ}$ 19012897, 2019, (in Italian).

[12] M.D.Zeiler, R. Fergus, "Visualizing and Understanding Convolutional Networks," Course CSCI 662 Advanced, Los Angeles: University of Southern California, 2013.

[13] J. Mockus, V. Tiesis, A. Zilinskas, "The application of Bayesian methods for seeking the extremum," Towards Global Optimization, n. 2, p. 117-129, 1978.

[14] D.R.Jones, "A taxonomy of global optimization methods based on response surfaces," Journal of Global Optimization, vol. 21(4), p. 345-383, 2001.

[15] R. Chiumeo, L. Garbero, L. Tenti, C. Gandolfi, M. de Nigris, "The voltage dip performance assessment of the Italian MV network through global indices", $21^{\text {si }}$ International Conference on Electricity Distribution, CIRED 2011, Frankfurt, paper 0886.

[16] CEI EN 61000-4-11 Compatibilità elettromagnetica (EMC) - Parte 4-11: Tecniche di prova e di misura - Prove d'immunità a buchi di tensione, brevi interruzioni e variazioni di tensione,» 2006.

[17] CEI EN 61000-4-34, «Compatibilità elettromagnetica (EMC) - Parte 4-34: Tecniche di prova e di misura - Prove d'immunità ai buchi di tensione e alle variazioni di tensione per le apparecchiature con corrente di ingresso superiore a 16 A per fase,» 2007. 